OPEN ACCESS

Edited by:

Xu Wang,

Affiliated Hospital of Jiangsu University,

China

Reviewed by:

Malgorzata Burek,

Julius Maximilian University of

Würzburg, Germany

Stephen B. Keysar

University of Colorado Anschutz

Medical Campus, United States

Yang Liu,

Hong Kong Polytechnic University,

China

*Correspondence:

Min Cui

cuimin@mail.hzau.edu.cn

Specialty section:

This article was submitted to Molecular and Cellular Oncology,

a section of the journal

Frontiers in Oncology

Received: 07 April 2021 Accepted: 31 May 2021

Published: 16 June 2021

Citation:

Wang $K$, Dong $S$, Higazy $D$,

Jin L, Zou Q, Chen H, Inayat A,

Hu S and Cui M (2021)

Inflammatory Environment Promotes the Adhesion of Tumor Cells to Brain

Microvascular Endothelial Cells.

Front. Oncol. 11:691771.

doi: $10.3389 /$ fonc.2021.691771

\section{Inflammatory Environment Promotes the Adhesion of Tumor Cells to Brain Microvascular Endothelial Cells}

\author{
Ke Wang 1,2,3,4, Shuang Dong ${ }^{5}$, Doaa Higazy ${ }^{1,2,3,4,6}$, Lijing Jin ${ }^{1,2,3,4}$, Qingcui Zou ${ }^{1,2,3,4}$, \\ Haowei Chen ${ }^{1,2,3,4}$, Aakif Inayat ${ }^{1,2,3,4}$, Sheng $\mathrm{Hu}^{5,7,8,9,10}$ and Min Cui ${ }^{1,2,3,4 *}$ \\ 1 State Key Laboratory of Agricultural Microbiology, College of Veterinary Medicine, Huazhong Agricultural University, \\ Wuhan, China, ${ }^{2}$ Key Laboratory of Preventive Veterinary Medicine in Hubei Province, The Cooperative Innovation Center for \\ Sustainable Pig Production, Wuhan, China, ${ }^{3}$ Key Laboratory of Development of Veterinary Diagnostic Products, Ministry of \\ Agriculture of the People's Republic of China, Wuhan, China, ${ }^{4}$ International Research Center for Animal Disease, Ministry of \\ Science and Technology of the People's Republic of China, Wuhan, China, ${ }^{5}$ Department of Medical Oncology, Hubei Cancer \\ Hospital, Tongji Medical College, Huazhong University of Science and Technology, Wuhan, China, ${ }^{6}$ Microbiology \\ Department, Faculty of Agriculture, Cairo University, Giza, Egypt, ${ }^{7}$ Hubei Provincial Cancer Center, Wuhan, China, \\ ${ }^{8}$ The Office of Hubei Provincial Cancer Prevention, Wuhan, China, ${ }^{9}$ The Cancer Quality Control Center of Hubei Province, \\ Wuhan, China, ${ }^{10}$ College of Health Science, Huazhong Agricultural University, Wuhan, China
}

Cancer patients usually suffer from unfavorable prognosis, particularly with the occurrence of brain metastasis of lung cancer. The key incident of brain metastasis initiation is crossing of blood-brain barrier (BBB) by cancer cells. Although preventing brain metastasis is a principal goal of cancer therapy, the cellular mechanisms and molecular regulators controlling the transmigration of cancer cells into the brain are still not clearly illustrated. We analyzed the mRNA expression profiles of metastatic brain tissues and TNF- $\alpha$ treated cancer cells to understand the changes in adhesion molecule expression during the tumor phase. To imitate the tumor microenvironment, an in vitro model was developed and the low or high metastatic potential lung tumor cells (A549 or H358) were cultured with the human brain microvascular endothelial cells (hBMECs) under TNF- $\alpha$ treatment. The analysis of online database indicated an altered expression for adhesion molecules and enrichment of their associated signaling pathways. TNF- $\alpha$ treatment activated hBMECs via up-regulating several adhesion molecules, including ICAM1, CD112, CD47, and JAM-C. Meanwhile, TNF- $\alpha$ induced an increased expression of adhesion molecule ligands such as ALCAM and CD6 in both A549 and H358. Moreover, the expression of adhesion molecules and the ligands were also increased both in A549or H358-hBMECs mixed culture system, which promoted tumor cells adhesion to endothelial cells. These results suggested that the enhanced interaction between tumor cells and brain microvascular endothelium might facilitate the incidence of metastatic brain tumors and further offer a better comprehension of brain metastasis prevention and treatment.

Keywords: tumor, brain metastasis, adhesion molecules, adhesion molecule ligands, human brain microvascular endothelial cells 


\section{INTRODUCTION}

The metastatic tumor is a pathological change that migrated from the original site to new tissues or organs. Among malignant tumors, metastasis was responsible for about $90 \%$ of cancer deaths $(1,2)$, which indicated a serious public health concern around the world. However, brain metastasis accounts for about $25 \%$ of all the metastatic cancers (3). As an organism's immune privilege area, the blood-brain barrier $(\mathrm{BBB})$ restricts the passage of medicine or immune cells to the brain, which might be one reason of patients to deteriorate and even to death upon the occurrence of brain metastasis.

Solid tumor cells undergo a series of complicated processes to spread to the circulatory system (4). The adhesion of the circulating tumor cells to the $\mathrm{BBB}$ is a critical step of brain metastasis (5). The BBB and blood-cerebrospinal fluid barrier separate the central nervous system from the circulatory system. The BBB is structurally composed of endothelial cells, pericytes, astrocyte endfeet, and neurons, while the endothelial cell tight junctions combined with pericytes form the basic structure of BBB (6). The endothelial cells play an important role in maintaining the $\mathrm{BBB}$, thus, the cell dysfunction always indicates a change of BBB permeability. Many diseases, such as neoplasia and viral infection, can induce the BBB breakdown (7, 8). However, brain metastasis often starts with endothelial cell activation. A recent study suggested that solid tumors changed the microenvironment and resulted in system inflammation (9). The induction of various inflammatory factors such as vascular endothelial growth factor C (VEGFC) could mediate endothelial cell activation during colorectal cancer invasion (10). Endothelial cell activation shows various changes, including the expression of adhesion molecules, cell morphologic alteration, cytokine production, and loss of barrier function. Previous studies indicated that inflammatory factors such as interleukin 8 (IL8 ), tumor necrosis factor $\alpha$ (TNF- $\alpha$ ), and VEGF mediated endothelial cell activation $(11,12)$, inducing the expression of adhesion molecules such as intercellular cell adhesion molecule 1 (ICAM-1), vascular endothelial cell adhesion molecule 1 (VCAM-1), and E-selectin, which were considered to promote leukocyte migration $(13,14)$. However, tumor cells may also spread to other organs under this mechanism.

Lung cancer is highly heterogeneous and known to spread to the brain in about $40 \%-50 \%$ of the metastatic cases, while breast cancer was about $15 \% \sim 20 \%(15,16)$. Tumor cells may create a chronic inflammatory microenvironment, producing various inflammatory factors such as CCL2, TNF- $\alpha$, and S100A8/A9 (17-19). TNF- $\alpha$ stimulated the expression of cluster of differentiation 62E (CD62E, E-selectin) on human brain endothelium (hCMEC/D3) which affected the brain metastasis of non-small cell lung cancer via the interaction of CD15s with CD62E (20). Furthermore, the co-culture of human lung

Abbreviations: hBMECs, human brain microvascular endothelial cells; BBB, blood-brain barrier; TNF- $\alpha$, tumor necrosis factor $\alpha$; VEGF, vascular endothelial growth factor; ICAM-1, intercellular cell adhesion molecule 1; ALCAM, activated leukocyte cell adhesion molecule; JAM-A, junctional adhesion molecule A. adenocarcinoma cell CL1-5 with human umbilical vein endothelial cells (HUVECs) altered the morphological structure of the latter (21). Thus, the inflammatory factors of the tumor microenvironment may be associated with the metastatic brain tumor.

The interaction of adhesion molecules and ligands is pivotal for tumor cells adherence to the endothelial cells, but the specific adhesion mechanism and the role of inflammatory factors in this process still need to be clarified. Through bioinformatics analysis, it was found that the adhesion molecules and their associated signaling pathways were up-regulated both in brain metastasis tumors and TNF- $\alpha$ stimulated cells. To verify these results, we used a mixed culture of lung tumor cells (A549 and H358) with brain microvascular endothelial cells (HBMECs) under TNF- $\alpha$ treatment to imitate the in vivo interaction. The A549 and H358 represented low and high metastatic potential lung tumor cells, respectively. A panel of genes associated with cell adhesion was screened with real-time PCR to assess the molecular event during the interaction systematically. The adhesion assay further indicated that the change of adhesion molecules and their ligands was associated with brain metastasis. These results may provide insights for preventing brain metastasis during tumor progression.

\section{MATERIALS AND METHODS}

\section{Publicly Available Data}

The transcriptomic data of three different experiments uploaded to the Gene Expression Omnibus (GEO) database were studied. The first experiment concerned primary lung cancer from nonsmall cell lung cancer patients with or without brain metastasis. The second experiment specifies the expression array profiling data of human endothelial cells. Human umbilical vein endothelial cells (HUVECs) were treated with various Staphylococcus aureus or TNF- $\alpha(100 \mathrm{ng} / \mathrm{ml}, 3.5 \mathrm{~h})$. Only the data from mock and TNF- $\alpha$ stimulated groups were analyzed in this study. The third experiment concluded the RNA sequencing data of TNF- $\alpha$ treated A549, and only the TNF- $\alpha$ treated (10 ng/ $\mathrm{ml}, 6 \mathrm{~h}$ ) or vehicle were extracted and considered in this research (22). The data for the three studies were downloaded from the GEO using the following accession numbers GSE126548, GSE82036, and GSM3538200, respectively.

\section{Cell Lines}

Human lung adenocarcinoma A549 and non-small cell lung carcinoma H358 cells (American Type Culture Collection, Manassas, VA) were amplified and stored in the laboratory. Cells were maintained in Dulbecco modified Eagle medium (DMEM) containing 10\% fetal bovine serum (Gibco, Grand Island, NY) and 1\% penicillin-streptomycin (Beyotime, China). The human brain microvascular endothelial cells (hBMECs) isolated from the children's cerebral microvessels were kindly provided by Prof. Xiangru Wang in the College of Veterinary Medicine, Huazhong Agricultural University, and originally obtained from John Hopkins University. hBMECs were 
cultured in RPMI 1640 medium supplemented with $10 \%$ fetal bovine serum, $1 \%$ penicillin-streptomycin, l-glutamine, sodium pyruvate, amino acid and vitamin in $37^{\circ} \mathrm{C}$ incubator with $5 \%$ $\mathrm{CO}_{2}$ and passaged every three days.

\section{Treatment of TNF- $\alpha$ on Various Cells}

Confluent hBMECs, A549 or H358 were seeded on 6-well plates at a concentration of $5 \times 10^{5}$ cells $/ \mathrm{ml}$. After overnight culture, hBMECs were stimulated with mouse recombinant TNF- $\alpha$ (R\&D Systems, Minneapolis, MN, USA) at a concentration of $40 \mathrm{ng} / \mathrm{ml}$, while A549 and H358 were stimulated with $5 \mathrm{ng} / \mathrm{ml}$ TNF- $\alpha$. For the mixed culture system, $5 \times 10^{5}$ cells $/ \mathrm{ml}$ of hBMECs were seeded overnight, then $5 \times 10^{5}$ cells/ml of A549 or H358 were added. The mixed cells were treated with DMEM medium containing $5 \mathrm{ng} / \mathrm{ml} \mathrm{TNF-} \alpha$. After $24 \mathrm{~h}$ or $48 \mathrm{~h}$, the supernatants were removed, and the cells were collected with TRIPure reagent (AidLab, China).

\section{Quantitative Real-Time PCR (qRT-PCR)}

Total cell mRNA was extracted according to the manufacturer's instructions of TRIPure reagent. Then the cDNA was synthesized with $5 \times$ All-In-One RT MasterMix (Applied Biological Materials, ABM, Canada). The qRT-PCR was performed with RealUniversal PreMix (TIANGEN, China) accordingly, and operated on a $\mathrm{ViiA}^{\mathrm{TM}} 7$ Real-Time PCR System (Applied Biosystems, Foster City, CA). A series of adhesion molecules and adhesion molecule ligands were screened with qRT-PCR. The data was analyzed by the comparative CT method as previously described (23). The primer pairs used in this research are as follows: ICAM1 (5'GCACATTGGTTGGCTATCTTCT-3' and 5'-GCCCGAAGC GTTTACTTTGA-3'), ICAM3 (5'-CGAGTTCTTGCAC AGGAACA-3' and $5^{\prime}$-CCTGAAGACGTACATTAAGGCC -3'), JAM-A (5'-GTGCCTTCAGCAACTCTTCC-3' and $5^{\prime}-$ GAGCCGATATCCGTTTGGTC-3'), JAM-B (5'-GTCTCCTT TGTCTACTATCAAC-3' and 5'-GGAGCCACTAATACTT CCAG-3'), JAM-C (5'-AAGGACGACTCTGGGCAGTA-3' and 5'-CGCCAATGTTCAGGTCATAG-3'), ALCAM (5'TCCTGCCGTCTGCTCTTCT- $3^{\prime}$ and $5^{\prime}$-TTCTGAGGTACG TCAAGTCGG-3'), CD6 (5'-GTGACCTGAAGGA GAATCTGC- $3^{\prime}$ and $5^{\prime}$-CCGGAGTGCAATCCTCTGG-3'), CD47 (5'-GGCAATGACGAAGGAGGTTA-3' and $5^{\prime}$-ATCC GGTGGTATGGATGAGA-3'), CD99 (5' - AACCCACCC AAACCGATGC-3' and 5' -TGAAAAGCTACCGGAG GAACTA-3'), CD112 (5'-GTCCTTCGTCTCTGCCAAGCA-3' and $5^{\prime}$-CACTGCGTGGATGACCAGCTG-3'), SLC44A1 (5'GGACCGTAGCTGCACAGAC- $3^{\prime}$ and $5^{\prime}$-GCCACAAATAA ATCCCATCCCA-3'), SIGLEC10 (5'-AAGGGACTCATCT CAACGGC-3' and 5'-CCGTCTCTTCGGTAGAATCTTCA$\left.3^{\prime}\right)$, and ITGB2 (5'-TGCGTCCTCTCTCAGGAGTG-3' and $5^{\prime}-$ GGTCCATGATGTCGTCAGCC-3').

\section{Cell Adhesion Analysis}

The hBMECs were seeded on a 24-well plate. Then the cells were treated with TNF- $\alpha$ ( $40 \mathrm{ng} / \mathrm{ml})$ or DMEM (control) for $24 \mathrm{~h}$ after the monolayers formed. Before adhesion assay, the A549 cells and H358 cells were suspended on PBS and labeled with CFSE tracer (Life Technologies, C34554, Eugene, USA) for 7 min of $3.75 \mu \mathrm{M}$, which was optimized according to manufacturer's instructions. The cells were washed with DMEM, and the labeled cells were visualized with the inverted fluorescence microscope (EVOS Cell Imaging Systems, Life Technologies) at the excitation/emission of $488 \mathrm{~nm} / 517 \mathrm{~nm}$. Then $200 \mu \mathrm{l}$ of A549 and $\mathrm{H} 358$ cells (containing $2 \times 10^{5}$ cells) were incubated with hBMECs for $2 \mathrm{~h}$. After three washes with PBS, the adherent tumor cells were observed and analyzed on the inverted fluorescence microscope.

\section{RNAseq Data Analysis}

The downloaded RNAseq data were analyzed. The reads were confirmed to quality by FastQC, mapped, and assembled to the human reference genome GRCh38 (hg38) using HISAT2 and featureCounts, respectively (24). The DESeq2 R package was used to obtain the differentially expressed genes (DEGs), with a $5 \% p$-adjusted value, and "apeglm" tool for log fold change shrinkage $(25,26)$. The $\mathrm{R}$ package clusterProfiler and DOSE were selected for the Gene ontology (GO) and Kyoto Encyclopedia of Genes and Genomes (KEGG) pathway analysis. While the figures were visualized using the $\mathrm{R}$ package enrichplot and ggplot2 (27).

\section{Statistical Analysis}

The data are representative results from three independent experiments and shown as means \pm SEMs. The significance was assessed with a two-tailed Student t-test or one-way ANOVA analysis, followed by Tukey's post hoc tests with the GraphPad Prism software (v7.0; GraphPad, La Jolla, CA).

\section{RESULTS}

\section{Targeted Gene Expression of Brain Metastasis Accompanied Lung Cancer Cells}

To explore brain metastasis's molecular events, RNA sequencing data were analyzed which were obtained from the GEO database for the primary tumor isolated from non-small cell lung cancer patients with brain metastasis $(\mathrm{BM}+)$ or without brain metastasis (BM-). The output results using the DESeq2 package indicated 488 differentially expressed genes (DEGs), among which 277 upregulated genes and 211 down-regulated genes for the primary lung tumor cells of the $\mathrm{BM}+$ patients over the $\mathrm{BM}$ - were observed. In the analysis, the DEGs were cut off to a $\log 2 \mathrm{FC}$ of 2 and a p-value was adjusted to less than 0.05 . The overrepresentation analysis (ORA) using clusterProfiler $\mathrm{R}$ package displayed the top 30 biological processes on a dotplot. The terms recording the highest gene ratio included the epidermic development $(24 / 297$, padj $=0.0007)$, epidermic cell differentiation $(18 / 297$, padj= 0.0083), skin development $(23 /$ 297 , padj $=0.0007)$, collagen-containing extracellular matrix $(17 /$ 319 , padj= 0.037), and keratinocyte differentiation $(15 / 297$, padj= 0.026) (Figure 1A). This result explains the active process of division, differentiation, and adhesion for lung 
A

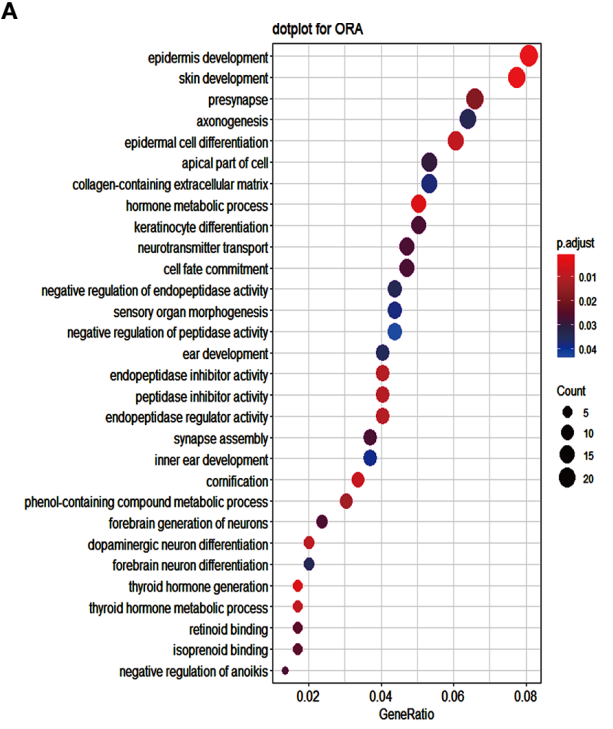

C

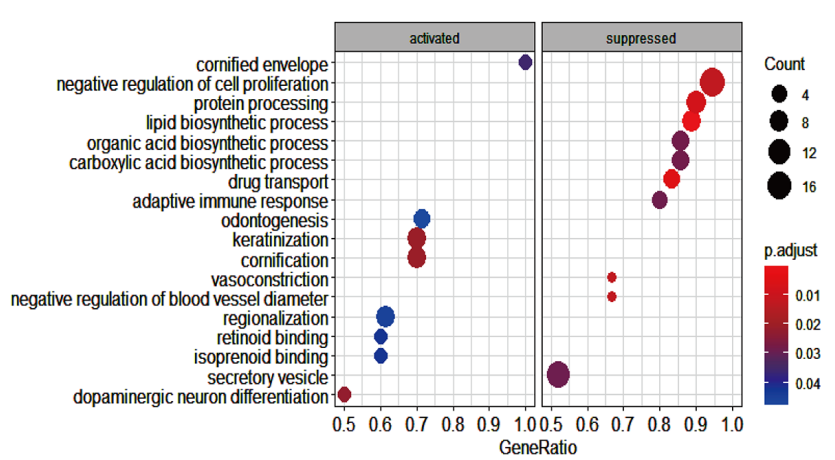

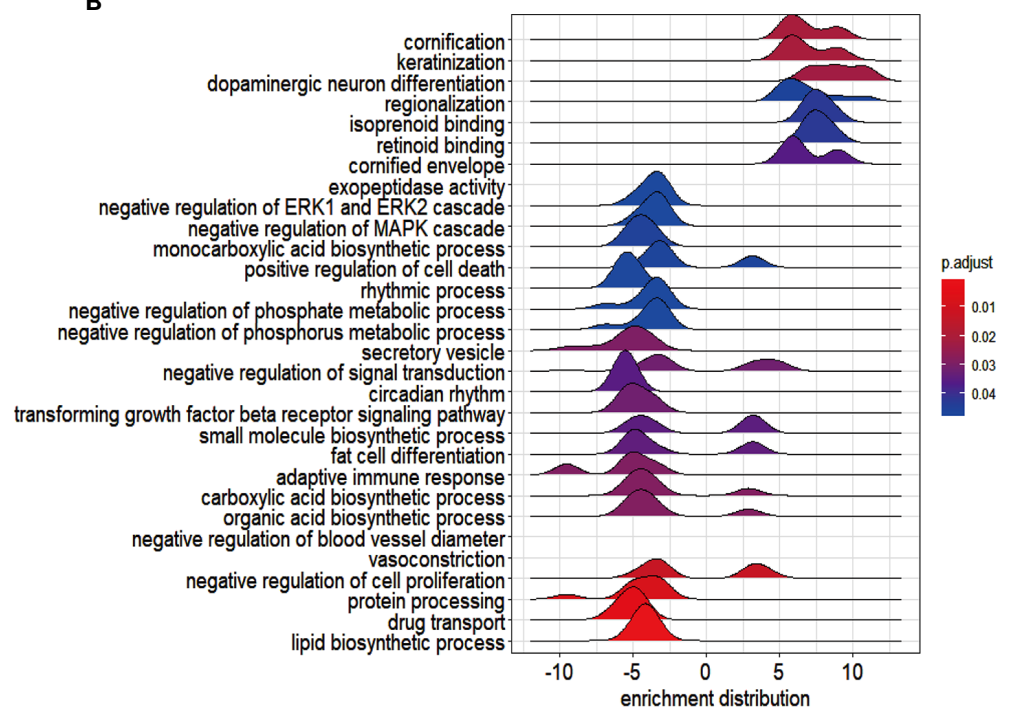

D

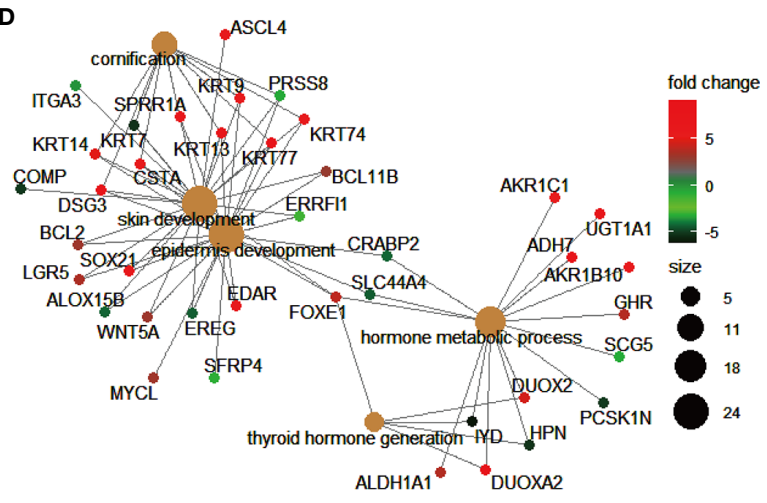

E

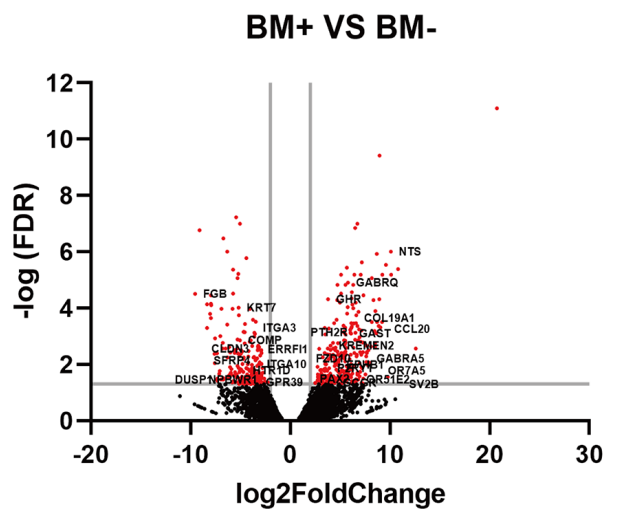

$\mathbf{F}$

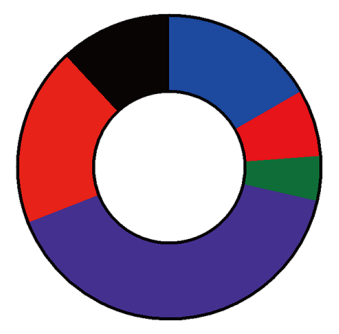

$\square$ EGFR1

$\square$ Cell adhesion molecules (CAMs)

Collagen biosynthesis and modifying enzymes

$\square$ Signaling by GPCR

Neuroactive ligand-receptor interaction

Wnt signaling pathway

FIGURE 1 | Transcriptome analysis summary of patients with brain metastasis (BM+) vs patients without brain metastasis (BM-). (A) Over representation analysis (ORA) for the enriched gene ontology (GO) terms obtained using the list of differentially expressed genes. The X-axis represented gene ratio, and the $\mathrm{Y}$-axis represented GO terms organized upon the number of genes giving rise to each term indicated in the size of dots and adjusted p-value shown in the color range (Blue to Red) of the dots. (B) Ridge plot of enriched terms visualizing the expression distributions of core enriched genes for GSEA enriched categories. The positive and negative value refers to whether each term was up- or down-regulated. (C) Gene ontology based GSEA dot plot indicates the top 10 activated and suppressed GO terms and plotted in the order of gene ratio. The $\mathrm{X}$-axis points to the gene ratio while the $\mathrm{Y}$-axis specifies the enriched terms, and $\mathrm{p}$-value is visualized in the dot color. (D) The cnetplot indicates the biological complexities among enriched GO terms and the overlapped genes in more than one term. The gray plots were the enriched pathways, while other plots represented genes. (E) Volcano plot exhibited the differentially expressed genes restricted to log2Foldchange (log2FC) of 1 and false discovery rate (FDR) ratio of 0.05. Red dots exhibit the significantly changed genes. (F) Pie chart of top 6 enriched GO terms that might be directly linked to the incidence of brain metastasis was presented. 
cancer accompanied by brain metastasis. The gene expression distribution was observed with a ridge plot that elucidated a positive regulation of cornification, keratinization, and neuron differentiation. Negative modulation of signaling cascades, such as extracellular signal-regulated kinases (ERKs) and mitogenactivated protein kinase (MAPK) signal (Figure 1B), suggested the initiation of inflammation. Consistent with Figures 1A, B, however, the gene set enrichment analysis (GSEA) revealed the top activated and inhibited GO terms, in which cornification and keratinization were significantly activated while there was an obvious suppression regarding the negative regulation of cell proliferation and vessel diameter (Figure 1C). The linkage of genes and associated pathways indicated that cornification, skin development, epidermal development, hormone metabolic process, and thyroid hormone pathways are interconnected and overlapped among their functional genes. The upregulated genes such as DSG3 and LGR5 might facilitate tumor metastasis, while the downregulation of ITGA3 changed the extracellular matrix and might promote tumor expansion (Figure 1D). The volcano plot showed significant DEGs. In $\mathrm{BM}+$ tumor cells CCL20 exhibited a dramatic increase (Figure 1E), while cytokines/chemokines such as CCL8, IL-6, and tumor necrosis factor superfamily members also showed an increasing trend. There was also a substantial downregulation of extracellular matrix (ITGA3 and ITGA10) and tight junction (CLDN3) transcripts (Figure 1E). Among the six biological processes of GO term enrichment, EGFR1, cell adhesion molecules, collagen, GPCR signaling, and Wnt signaling were possibly associated with tumor brain metastasis (Figure 1F). These results suggested that the change in cell division and cell differentiation might be associated with tumor progression. However, the ultimate suppression of the extracellular matrix and inflammation activation were probably responsible for tumor brain metastasis.

\section{TNF- $\alpha$ Induced the Expression of Adhesion Molecules and Adhesion Molecule Ligands on Human Endothelial Cells and Tumor Cells}

To figure out the role of microenvironment inflammatory factor on endothelial cells and tumor cells, the data of TNF- $\alpha$ treated human umbilical vein endothelial cells (HUVECs) and TNF- $\alpha$ treated A549 data from the GEO database were analyzed. The TNF- $\alpha$ treated HUVECs induced the upregulation of adhesion molecules, including ICAM1, VCAM1, CLEC2D, JAM2 (JAM-B), $C D 47$, and $A L C A M$ (Figure 2A). On the other hand, TNF- $\alpha$ stimulated A549 enhanced the mRNA expression of both adhesion molecules and adhesion molecule ligands such as SELE, ICAM1, ICAM4, ITGAM, SL-1, SELL, SIGLEC10, and SIGLEC11 (Figure 2B). Moreover, TNF- $\alpha$ treatment modulated the expression of migration-related genes on A549, which included MMP10, EGFR, COX2 and EREG (Figure 2B). It revealed that adhesion molecules and their ligands on endothelial cells and tumor cells induced by TNF- $\alpha$ might promote tumor adhesion.

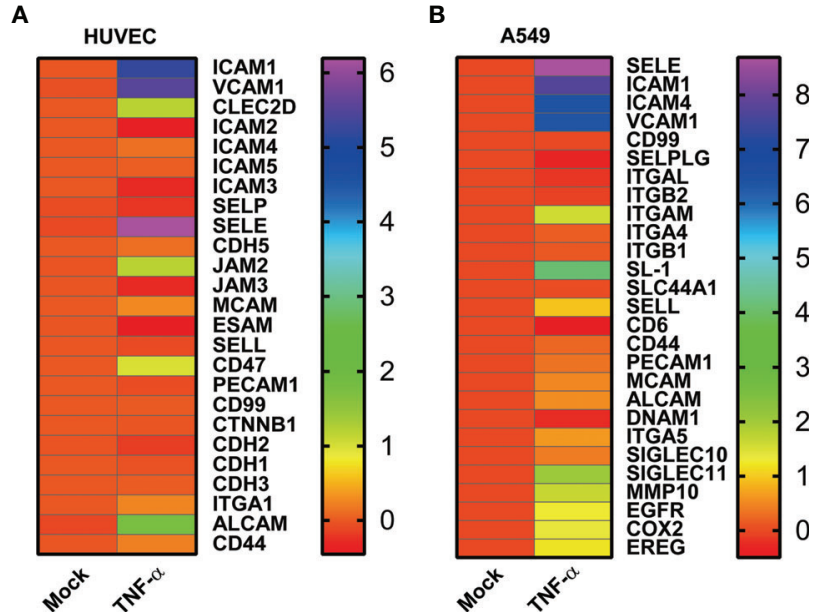

FIGURE 2 | High throughput data analysis of HUVECs and A549 cells. (A) The heatmap indicates the mRNA adhesion molecules on HUVECs induced by TNF- $\alpha$, and (B) adhesion associated genes on A549 determined in the presence of TNF- $\alpha$. Each group (Mock and TNF- $\alpha$ ) represented the average value of three duplicates. Data were shown as log2 fold change to mock.

\section{TNF- $\alpha$ Induced Human Brain Microvascular Endothelial Cells (hBMECs) Activation}

In vascular disease, numerous inflammatory cytokines and other factors are associated with endothelial cells activation (28). To identify whether cytokines could activate hBMECs, we applied a DMEM culture medium supplemented with $40 \mathrm{ng} / \mathrm{ml} \mathrm{TNF-} \alpha$ to the cultured cells. The mRNA levels of adhesion molecule genes were quantified with qRT-PCR. The expression of ICAM3 was significantly decreased at $24 \mathrm{~h}$ but increased at $48 \mathrm{~h}$ compared to control after TNF- $\alpha$ treatment (Figure 3A). CD112 (nectin-2) showed no changes at $24 \mathrm{~h}$, but significantly up-regulated at $48 \mathrm{~h}$ (Figure 3B). However, the expression of CD47 and JAM-C (junctional adhesion molecule $\mathrm{C}$, also known as JAM3) began to elevate at $24 \mathrm{~h}$ and markedly increased at $48 \mathrm{~h}$ after TNF- $\alpha$ treatment (Figures 3C, D). These results suggested that variation in microenvironment mediated the activation of endothelial cells, and further exhibited a significant expression change of the adhesion molecules.

\section{Alteration of the Microenvironment Led to Upregulation of Adhesion Molecule Ligands on Tumor Cells}

Inflammatory cytokines could induce cell-surface ICAM1 expression on tumor cells as previously described $(29,30)$. Human lung adenocarcinoma A549 cells were isolated from the solid tumors (31), while the non-small cell lung carcinoma H358 cells were originated from a metastatic tissue, the cells of which had low and high metastatic potential, respectively. Interaction of cell adhesion molecules and the ligands is a critical step of tumor 
A

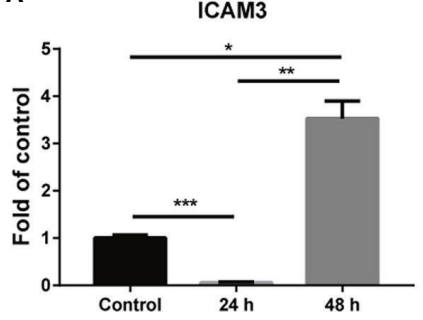

B

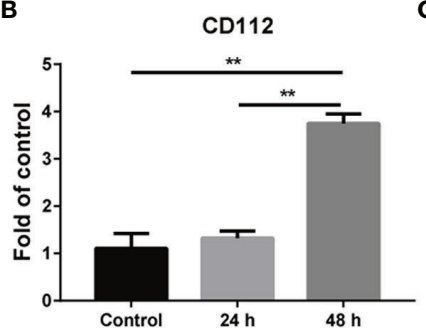

C

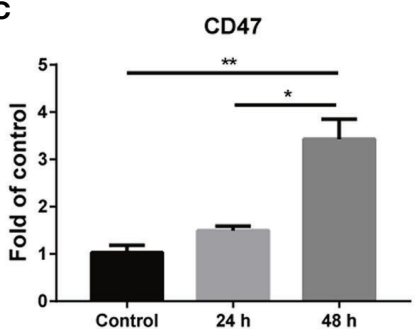

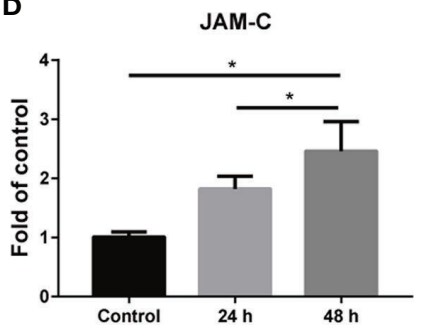

FIGURE 3 | Activation of hBMECs after TNF- $\alpha$ treatment. Confluent hBMECs monolayers were treated with DMEM or DMEM containing $40 \mathrm{ng} / \mathrm{ml}$ TNF- $\alpha$. Total RNA was collected at $24 \mathrm{~h}$ and $48 \mathrm{~h}$. The quantification of cell adhesion molecule genes was detected with real-time PCR (A-D). The fold change of treated cell adhesion molecule genes compared to the control over time was shown after normalized to $\beta$-actin. The data are representative of three independent experiments and expressed as mean \pm SEMs. ${ }^{*} p<0.05 ;{ }^{\star \star} p<0.01 ;{ }^{* \star} p<0.001$.

metastasis (2). Hence, the expression of adhesion molecule ligands in A549 and $\mathrm{H} 358$ were detected in the presence of $5 \mathrm{ng} / \mathrm{ml}$ TNF- $\alpha$. The mRNA level of ALCAM (activated leukocyte cell adhesion molecule), CD6, and SIGLEC10 (sialic acid-binding Ig-like lectin 10) on A549 cells were substantially elevated at $24 \mathrm{~h}$ and peaked at $48 \mathrm{~h}$ compared to the control (Figures 4A, C, D). However, the SLC44A1 (encoding CD92) dramatically increased on the mRNA level at $48 \mathrm{~h}$ but not at $24 \mathrm{~h}$ (Figure 4B). ALCAM expression on H358 markedly increased at $24 \mathrm{~h}$ and $48 \mathrm{~h}$ after treated with TNF- $\alpha$, which was consistent with the result of ALCAM on A549 (Figure 4E). Similarly, the expression of CD6, CD99 and ITGB2 (encoding a subunit of LFA-1) on H358 cells showed no or slight change at $24 \mathrm{~h}$ but significantly up-regulated at $48 \mathrm{~h}$ compared with the control group (Figures $\mathbf{4 F}-\mathbf{H}$ ). These results indicated that inflammatory microenvironment could (such as TNF- $\alpha$ ) mediate both low and high metastatic potential tumor cells expressing adhesion molecule ligands.

\section{Mixed Culture of A549 With hBMECs Induced the Expression of Adhesion Molecules and Adhesion Molecule Ligands in the Presence of TNF- $\alpha$}

To imitate the internal tumor migration environment, A549 cells and hBMECs were incubated on a culture medium supplemented with $5 \mathrm{ng} / \mathrm{ml}$ TNF- $\alpha$. As receptors and ligands of adhesion molecules mutually interact, the expression of adhesion molecules and their ligands was determined on

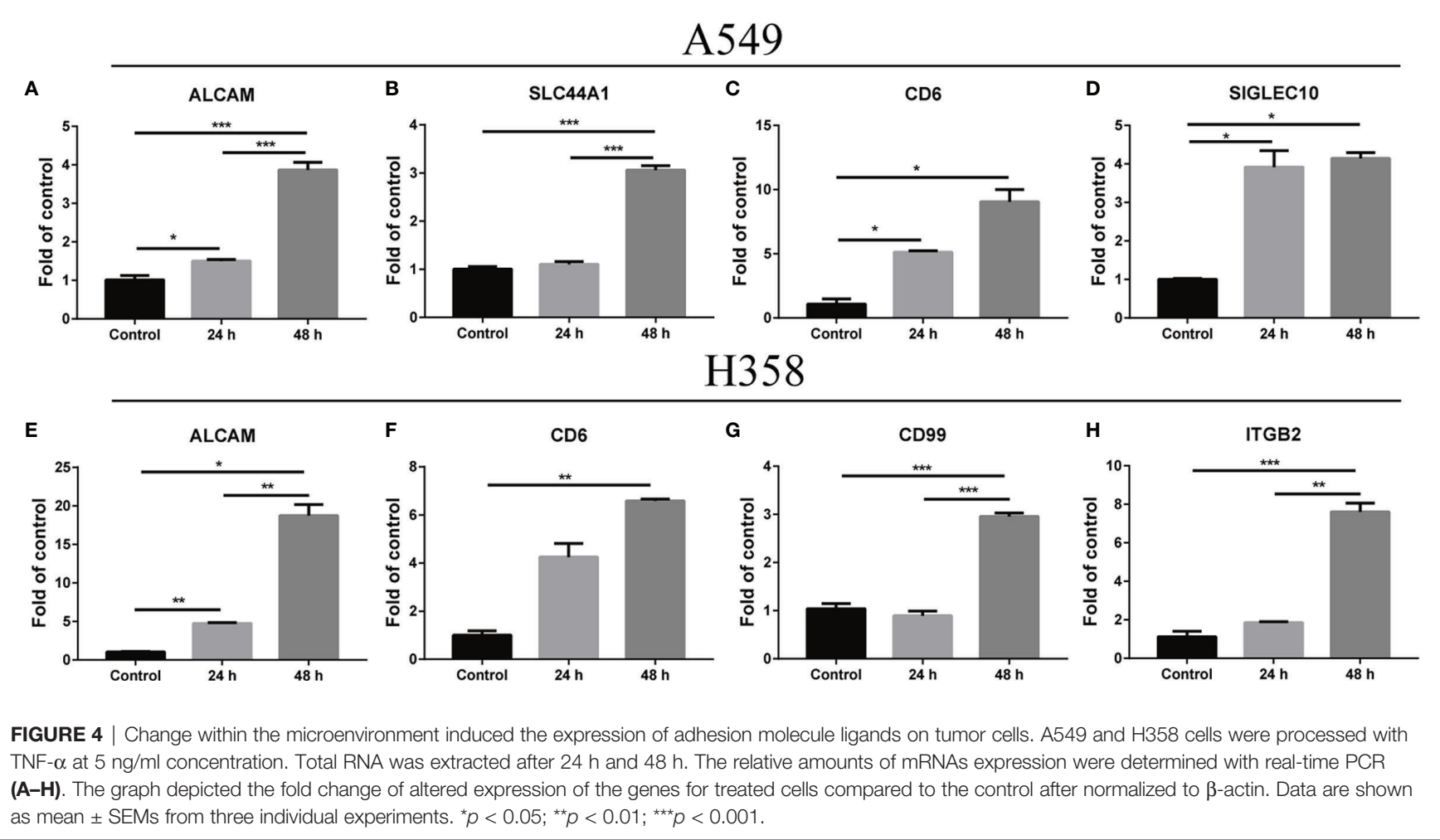


mixed cells. The expression of CD6 was memorably elevated both at $24 \mathrm{~h}$ and $48 \mathrm{~h}$ after TNF- $\alpha$ treatment (Figure 5A). The mRNA of ICAM1, JAM-B (JAM2) and SIGLEC10 showed no marked change at $24 \mathrm{~h}$ but significantly increased at $48 \mathrm{~h}$ compared to the control (Figures 5B-D). Taken together, it is suggested that TNF- $\alpha$ mediated the expression of adhesion molecules and their ligands after incubation of tumor cells with endothelial cells.

\section{Mixed Culture of H358 With hBMECs Mediated Adhesion Molecules and Adhesion Molecule Ligands mRNA Expression Under TNF- $\alpha$ Treatment}

To demonstrate whether high metastatic potential tumor cells could interact with endothelial cells, lung carcinoma H358 cells were incubated with hBMECs in a culture medium containing 5 $\mathrm{ng} / \mathrm{ml}$ TNF- $\alpha$. The expression of adhesion molecules and their ligands of mixed cells were quantified with real-time PCR. CD47 and CD99 only showed a substantial increase at mRNA level after TNF- $\alpha$ treated for $48 \mathrm{~h}$ (Figures 6A, B). Both ICAM3 and JAM-A (JAM1) were dramatically elevated at $24 \mathrm{~h}$ and $48 \mathrm{~h}$ compared to control after treated with TNF- $\alpha$ (Figures 6C, D). Surprisingly, the ICAM3 displayed a declining trend from $24 \mathrm{~h}$ to $48 \mathrm{~h}$. These results, consistent with previous data, indicated that the microenvironment changed the adhesion molecules' and their ligands' expression during tumor-endothelial cell interaction.

\section{Microenvironment TNF- $\alpha$ Enhanced Adhesion of Tumor Cells to Endothelial Cells}

Since TNF- $\alpha$ up-regulated adhesion molecules and their ligands on tumor cells and endothelial cells, we wondered whether this inflammatory factor was associated with tumor cells adhesion. CFSE was used to trace the adhesion of A549 and H358 cells, which showed almost 100\% labeling efficiency (Figure 7A). Adhesion assay demonstrated that both A549 and H358 could adhere to hBMECs to some extent (Figure 7B). Once stimulated with TNF$\alpha$, the adherent A549 and $\mathrm{H} 358$ cells increased dramatically, which were $253 \%$ and $357 \%$ (Figures 7B, C), respectively. By linking the current result with previous experiments, it is suggested that the overexpressed adhesion molecules and their ligands on endothelial cells and tumor cells by microenvironment TNF- $\alpha$ were responsible for tumor metastasis.

\section{DISCUSSION}

Tumor progression including growth, invasion, and metastasis, is closely related to the tumor microenvironment (32).
A

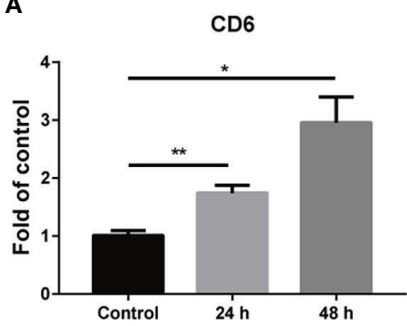

B

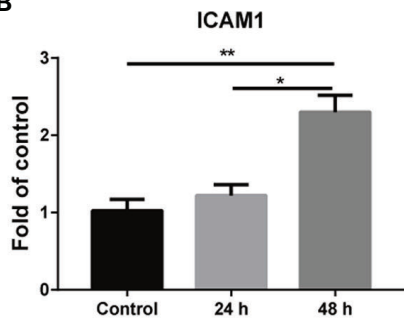

C

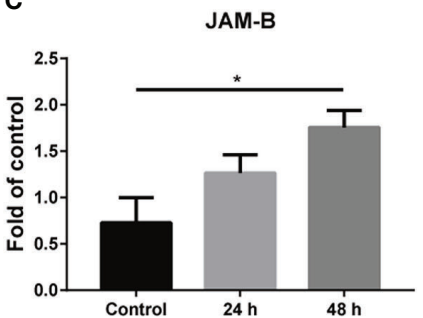

D

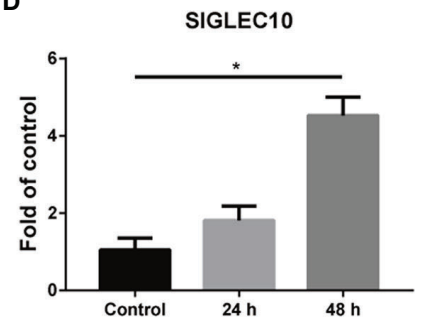

FIGURE 5 | Mixed culture of A549 with hBMECs up-regulated the expression of adhesion molecules and adhesion molecule ligands. hBMECs were seeded overnight, and then A549 cells were added at a ratio of 1:1 under $5 \mathrm{ng} / \mathrm{ml} \mathrm{TNF-} \alpha$ treatment. Mixed cells were collected for RNA isolation at $24 \mathrm{~h}$ and $48 \mathrm{~h}$. The altered gene expression of adhesion molecule and adhesion molecule ligand mRNAs were detected with real-time PCR (A-D). The data are representative results from three independent experiment and displayed as mean \pm SEMs. ${ }^{*} p<0.05 ;{ }^{* *} p<0.01$.

A

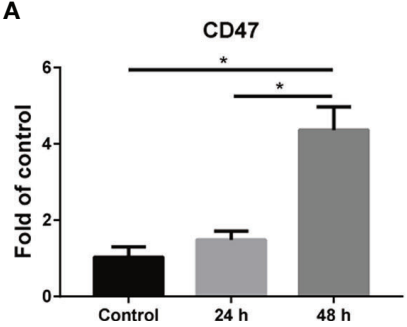

B

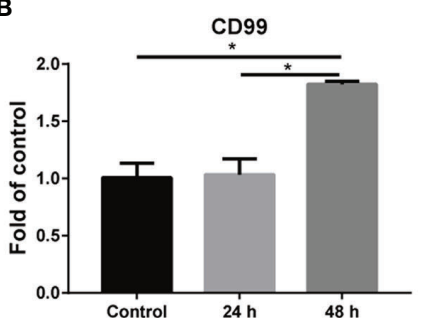

C

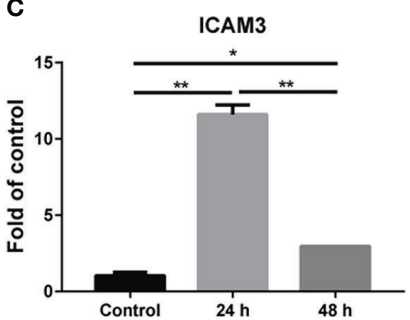

D

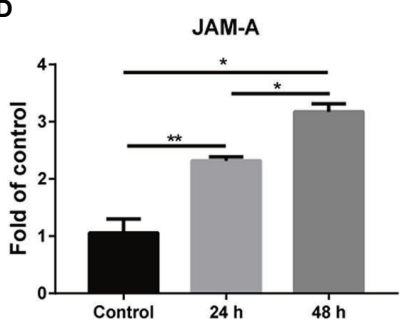

FIGURE 6 | Mixed culture of H358 with hBMECs mediated the upregulation of adhesion molecules and adhesion molecule ligands. hBMECs were seeded overnight, and then $\mathrm{H} 358$ cells were added at a ratio of 1:1. The mixed cells were treated with $5 \mathrm{ng} / \mathrm{ml} \mathrm{TNF}-\alpha$, and total RNA was extracted at $24 \mathrm{~h}$ and $48 \mathrm{~h}$. Relative expression of mRNAs was determined with real-time PCR (A-D). The data are representative results from three independent experiment and shown as mean \pm SEMs. ${ }^{*} p<0.05 ;{ }^{* \star} p<0.01$. 
A

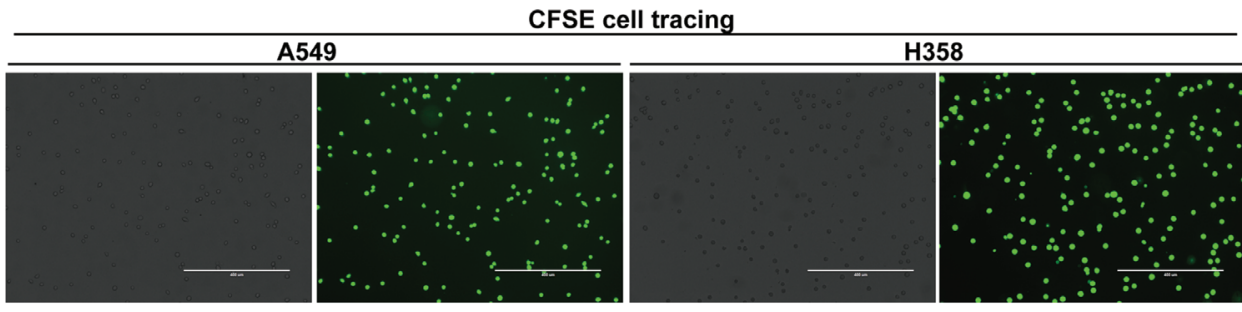

B
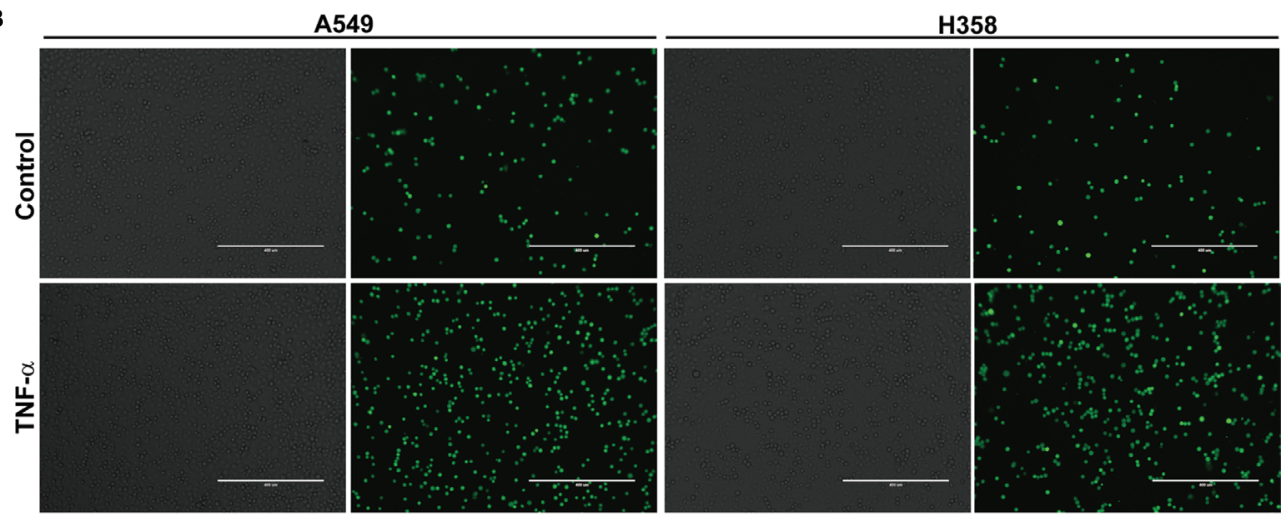

C
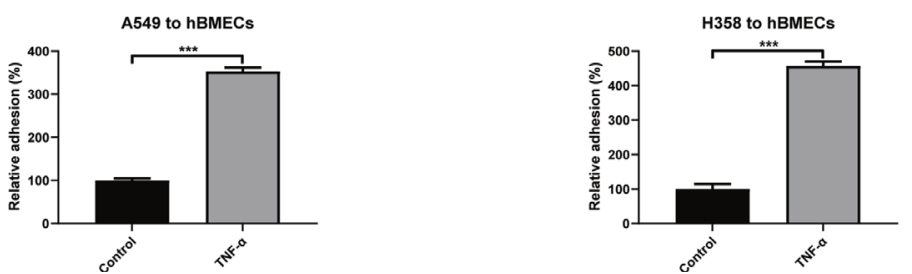

FIGURE 7 | TNF- $\alpha$ promoted adhesion of tumor cells to endothelial cells. (A) A549 and H358 cells were labeled with CFSE dye, and the staining efficiency was evaluated with an inverted fluorescence Microscope. (B) Adhesion of A549 and H358 cells to hBMECs was observed on an inverted fluorescence Microscope after hBMECs treated with DMEM (control) or TNF- $\alpha$ (40 ng/ml). (C) The relative adherent cell numbers in (B) were quantified. One representative image of three independent experiments were shown in this figure. The left and right panels in each cell were the bright field image and fluorescent image, respectively. Scale bar was $400 \mu \mathrm{m} .{ }^{* \star *} p<0.001$.

The tumor microenvironment consists of non-tumor cells, such as endothelial cells, tumor-associated macrophages (TAMs), myeloid-derived suppressor cells (MDSCs), tumor-associated neutrophils (TANs), and various other cells, as well as cytokine interferon $\alpha$ (IFN- $\alpha)$, TNF, IL-1 $\beta$ and IL-6 to sustain an inflammatory environment (33). TAMs served as a potential anti-cancer therapeutic target because of its protumor functions (34). On the other hand, TAMs produced TNF- $\alpha$ both in vivo and in vitro, indicating that TAMs affected systemic inflammatory state (35). To mimic the in vivo tumor metastasis, a mixed culture model was utilized to incubate the tumor cells with hBMECs under TNF- $\alpha$ treatment. Obviously, there is an increased expression of adhesion molecules and their ligands in the mixed culture system with TNF- $\alpha$ treatment. It was speculated that these changes played a critical role in tumor brain metastasis during tumor progression.

Diverse humoral factors, including cytokines, lipopolysaccharides, polar phospholipids, homocysteine, and viral infection stimulated endothelial activation $(14,36-38)$. The activated cells were accompanied by adhesion molecules VCAM-
1, ICAM-1 and E-selectin expression (39). IL-1 $\alpha$, IL-1 $\beta$, IL-4 and TNF- $\alpha$ induced endothelial activation by mediating VCAM-1 production and promoting monocyte adhesion to endothelial monolayers, but nitric oxide limited this process (40). These studies suggested that endothelial activation was possibly involved in tumor brain metastasis. Indeed, TNF- $\alpha$ promoted adhesion of lung cancer cells to brain endothelium via a CD15s-CD62E manner, while blocking CD15s decreased this process (20). This result is consistent with our results. Moreover, the mixed culture of cancer cells with endothelial cells promoted angiogenesis and increased migration-related genes, indicating a boost of metastasis (21).

The interaction of adhesion molecules and adhesion molecule ligands widely existed during adhesion and migration of leukocytes. It was reported that ICAM-1- lymphocyte function-associated antigen 1 (LFA-1) interaction was essential for $\mathrm{T}$ or $\mathrm{NK}$ cells adhering to the target cells (41). ALCAM-CD6 mediated T cells adhering to dendritic cells and induced T cell proliferation (42). The specific interactions between adhesion molecules and their ligands were important for immune cell migration and activation. Similarly, the interaction of mutual adhesion molecules had multiple 


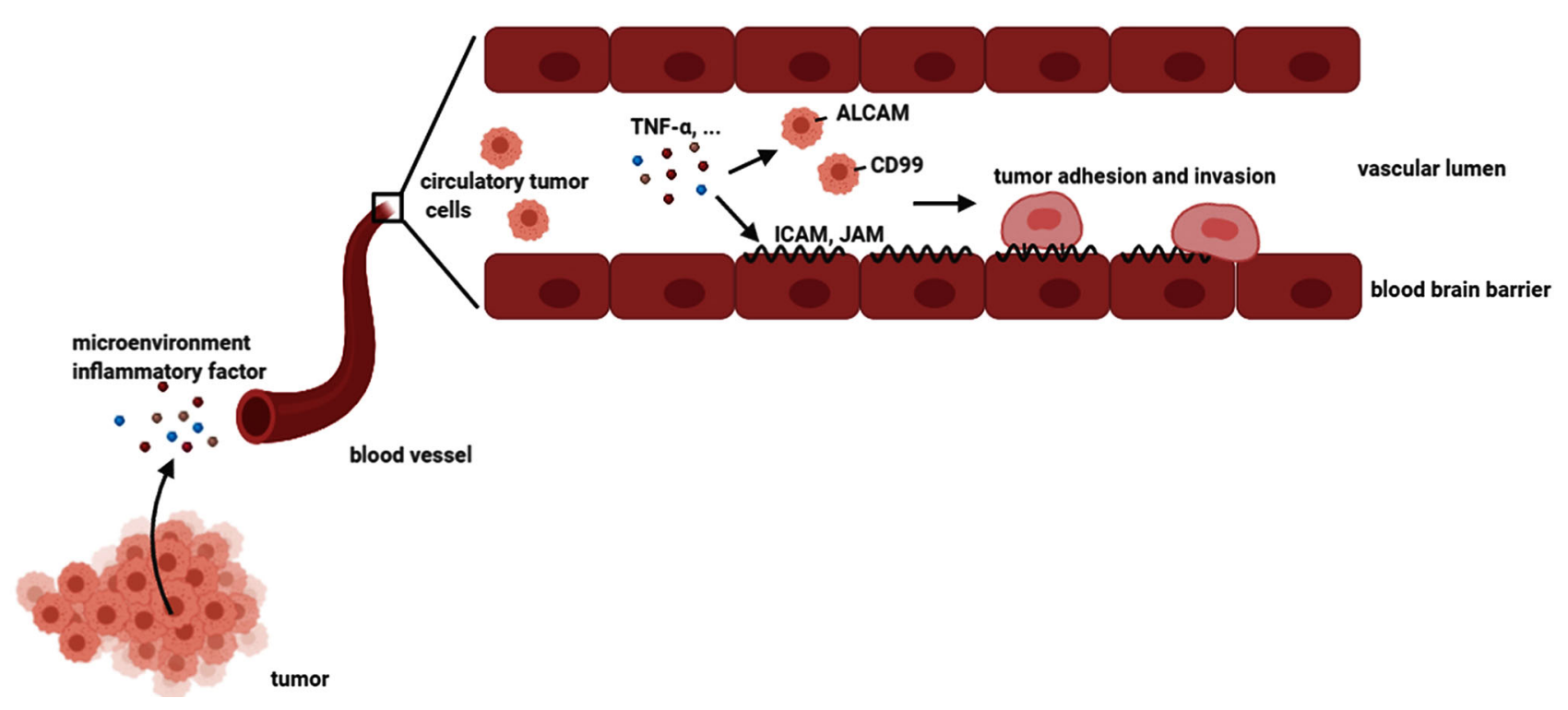

FIGURE 8 | A schemata of tumor brain metastasis. The tumor cells could secrete many inflammatory factors. Some tumor cells could migrate to the circulatory system, which is called circulating tumor cells. Microenvironment inflammatory factors such as TNF- $\alpha$ could stimulate both brain endothelial cells and tumor cells, resulting in the upregulation of adhesion molecules and their ligands on endothelial cells and tumor cells, respectively. Consequently, the tumor cells adhered and invaded to endothelial cells, leading to brain metastasis.

functions. ICAM-1-ICAM-1 homophilic interaction was associated with breast cancer metastatic amplification (43), while ALCAMALCAM interaction was an assistant for T cell adhering to dendritic cells (42). The interaction of identical adhesion molecules might become favorable in some pathological processes, such as tumor metastasis. In this study, we found an altered expression of adhesion molecules on endothelial cells and their ligands on tumor cells in the presence of TNF- $\alpha$. This interaction might facilitate tumorendotheliocyte adhesion, rolling, crossing, and contribute to tumor brain metastasis.

The specific mechanism of how tumor cells intruding into the central nervous system was ambiguous, but the current research could provide some cues. The possible routes of metastasis included the seed and soil hypothesis (44), the blood-brain barrier theory (45), and the tumor stem cell theory (46). However, the BBB theory was exquisite and much more attractive. Breast cancer cells infiltrated into the brain using a surface glycosylation $\alpha 2,6$ sialyltransferase ST6GALNAC5 to interact with and cross the BBB (47). Acute lymphoblastic leukemia cells (ALLs) invaded the central nervous system via crawling along the emissary vessels of subarachnoid space in an integrin- $\alpha 6$-laminin manner without breaching the BBB (48). JAM-A was believed to cause unfavorable prognosis in breast cancer patients via the ligand $\beta 1$ integrin (49). However, Naik et al. demonstrated that JAM-A downregulation induced breast cancer cells to spread from the primary tumor site (50), which might due to the different tumor models and dynamic expression in different progression stages. The decrease of adhesion molecules induced tumor cells to disperse from the original site. However, upregulation of adhesion molecules enhanced tumor cells adhesion to endothelial cells and promoted tumor metastasis, which was generally presented in this study.
These findings attracted our attention to the effect of adhesion molecules on tumor brain metastasis, as previously mentioned (5). As shown in Figure 8, our results suggested that the microenvironment TNF- $\alpha$ activated hBMECs endothelial cells, induced adhesion molecules expression, and mediated the expression of adhesion molecule ligands on tumor cells. It was indicated that TNF- $\alpha$ up-regulated adhesion molecules and their ligands in the mixed culture system and facilitated the interaction of tumor cells with endothelial cells that might further promote brain metastasis. Future research may focus on identifying the specific molecules that are associated with brain metastasis. Prospectively, a combination of inflammatory factors (such as TNF- $\alpha$ ) and adhesion molecules (such as ICAM1 and ALCAM) neutralizing antibodies might be a useful adjuvant therapy to prevent tumor metastasis in the process of tumor treatment.

\section{DATA AVAILABILITY STATEMENT}

The datasets presented in this study can be found in online repositories. The names of the repository/repositories and accession number(s) can be found in the article/ supplementary material.

\section{AUTHOR CONTRIBUTIONS}

Experiment was design by KW, MC, SD and LJ. The experiments of this study were performed by LJ, QZ and HC. The profile data 
were analyzed by $\mathrm{DH}$, and the other data were analyzed by $\mathrm{LJ}$, and KW. KW wrote this manuscript, while MC, AI, SD and SH kindly revised it. All authors have full access to this data and complete the responsibility part. All authors contributed to the article and approved the submitted version.

\section{FUNDING}

The authors are grateful for the financial support provided by the National Program on Key Research Project of China

\section{REFERENCES}

1. Chaffer CL, Weinberg RA. A Perspective on Cancer Cell Metastasis. Science (2011) 331:1559-64. doi: 10.1126/science.1203543

2. Guan X. Cancer Metastases: Challenges and Opportunities. Acta Pharm Sin B (2015) 5:402-18. doi: 10.1016/j.apsb.2015.07.005

3. Svokos KA, Salhia B, Toms SA. Molecular Biology of Brain Metastasis. Int J Mol Sci (2014) 15:9519-30. doi: 10.3390/ijms15069519

4. Berghoff AS, Preusser M. The Inflammatory Microenvironment in Brain Metastases: Potential Treatment Target? Chin Clin Oncol (2015) 4:21. doi: 10.3978/j.issn.2304-3865.2015.06.03

5. Achrol AS, Rennert RC, Anders C, Soffietti R, Ahluwalia MS, Nayak L, et al. Brain Metastases. Nat Rev Dis Primers (2019) 5:5. doi: 10.1038/s41572-0180055-y

6. Sweeney MD, Sagare AP, Zlokovic BV. Blood-Brain Barrier Breakdown in Alzheimer Disease and Other Neurodegenerative Disorders. Nat Rev Neurol (2018) 14:133-50. doi: 10.1038/nrneurol.2017.188

7. Ballabh P, Braun A, Nedergaard M. The Blood-Brain Barrier: An Overview: Structure, Regulation, and Clinical Implications. Neurobiol Dis (2004) 16:113. doi: 10.1016/j.nbd.2003.12.016

8. Wang K, Wang H, Lou W, Ma L, Li Y, Zhang N, et al. Ip-10 Promotes BloodBrain Barrier Damage by Inducing Tumor Necrosis Factor Alpha Production in Japanese Encephalitis. Front Immunol (2018) 9:1148. doi: 10.3389/ fimmu.2018.01148

9. Demers M, Suidan GL, Andrews N, Martinod K, Cabral JE, Wagner DD. Solid Peripheral Tumor Leads to Systemic Inflammation, Astrocyte Activation and Signs of Behavioral Despair in Mice. PloS One (2018) 13:e0207241. doi: 10.1371/journal.pone.0207241

10. Tacconi C, Correale C, Gandelli A, Spinelli A, Dejana E, D'alessio S, et al. Vascular Endothelial Growth Factor C Disrupts the Endothelial Lymphatic Barrier to Promote Colorectal Cancer Invasion. Gastroenterology (2015) 148:1438-51.e1438. doi: 10.1053/j.gastro.2015.03.005

11. Mechtcheriakova D, Schabbauer G, Lucerna M, Clauss M, De Martin R, Binder BR, et al. Specificity, Diversity, and Convergence in VEGF and TNF-Alpha Signaling Events Leading to Tissue Factor Up-Regulation Via EGR-1 in Endothelial Cells. FASEB J (2001) 15:230-42. doi: 10.1096/fj.00$0247 \mathrm{com}$

12. Rondaij MG, Bierings R, Kragt A, Van Mourik JA, Voorberg J. Dynamics and Plasticity of Weibel-Palade Bodies in Endothelial Cells. Arterioscler Thromb Vasc Biol (2006) 26:1002-7. doi: 10.1161/01.ATV.0000209501.56852.6c

13. Adams DH, Shaw S. Leucocyte-Endothelial Interactions and Regulation of Leucocyte Migration. Lancet (1994) 343:831-6. doi: 10.1016/S0140-6736(94) 92029-X

14. Li F, Wang Y, Yu L, Cao S, Wang K, Yuan J, et al. Viral Infection of the Central Nervous System and Neuroinflammation Precede Blood-Brain Barrier Disruption During Japanese Encephalitis Virus Infection. J Virol (2015) 89:5602-14. doi: 10.1128/JVI.00143-15

15. Barnholtz-Sloan JS, Sloan AE, Davis FG, Vigneau FD, Lai P, Sawaya RE. Incidence Proportions of Brain Metastases in Patients Diagnoseto 2001) in the Metropolitan Detroit Cancer Surveillance System. J Clin Oncol (2004) 22:2865-72. doi: 10.1200/JCO.2004.12.149

16. De Sousa VML, Carvalho L. Heterogeneity in Lung Cancer. Pathobiology (2018) 85:96-107. doi: 10.1159/000487440
(2016YFD0500406), the National Natural Sciences Foundation of China (Grant No. 31172294), the Fundamental Research Funds for the Central Universities Grant 2662018PY016, and Natural Science Foundation of Hubei Province (2019CFA010).

\section{ACKNOWLEDGMENTS}

We thank Dr. Xiangru Wang (Huazhong Agricultural University) who generously provided hBMECs cells in this research.

17. Hiratsuka S, Watanabe A, Sakurai Y, Akashi-Takamura S, Ishibashi S, Miyake K, et al. The S100A8-Serum Amyloid A3-TLR4 Paracrine Cascade Establishes a Pre-Metastatic Phase. Nat Cell Biol (2008) 10:1349-55. doi: 10.1038/ncb1794

18. Kim S, Takahashi H, Lin WW, Descargues P, Grivennikov S, Kim Y, et al. Carcinoma-Produced Factors Activate Myeloid Cells Through TLR2 to Stimulate Metastasis. Nature (2009) 457:102-6. doi: 10.1038/nature07623

19. Said N, Sanchez-Carbayo M, Smith SC, Theodorescu D. RhoGDI2 Suppresses Lung Metastasis in Mice by Reducing Tumor Versican Expression and Macrophage Infiltration. J Clin Invest (2012) 122:1503-18. doi: 10.1172/ JCI61392

20. Jassam SA, Maherally Z, Smith JR, Ashkan K, Roncaroli F, Fillmore HL, et al. Cd15s/Cd62e Interaction Mediates the Adhesion of Non-Small Cell Lung Cancer Cells on Brain Endothelial Cells: Implications for Cerebral Metastasis. Int J Mol Sci (2017) 18:1474. doi: 10.3390/ijms18071474

21. Cheng HW, Chen YF, Wong JM, Weng CW, Chen HY, Yu SL, et al. Cancer Cells Increase Endothelial Cell Tube Formation and Survival by Activating the PI3K/Akt Signalling Pathway. J Exp Clin Cancer Res (2017) 36:27. doi: 10.1186/s13046-017-0495-3

22. Manjur A, Lempiainen JK, Malinen M, Palvimo JJ, Niskanen EA. IRF2BP2 Modulates the Crosstalk Between Glucocorticoid and TNF Signaling. J Steroid Biochem Mol Biol (2019) 192:105382. doi: 10.1016/j.jsbmb.2019.105382

23. Schmittgen TD, Livak KJ. Analyzing Real-Time PCR Data by the Comparative C(T) Method. Nat Protoc (2008) 3:1101-8. doi: 10.1038/nprot.2008.73

24. Kim D, Langmead B, Salzberg SL. HISAT: A Fast Spliced Aligner With Low Memory Requirements. Nat Methods (2015) 12:357-60. doi: 10.1038/ nmeth.3317

25. Love MI, Huber W, Anders S. Moderated Estimation of Fold Change and Dispersion for RNA-seq Data With Deseq2. Genome Biol (2014) 15:550. doi: 10.1186/s13059-014-0550-8

26. Zhu A, Ibrahim JG, Love MI. Heavy-Tailed Prior Distributions for Sequence Count Data: Removing the Noise and Preserving Large Differences. Bioinformatics (2019) 35:2084-92. doi: 10.1093/bioinformatics/bty895

27. Wickham H. ggplot2: Elegant Graphics for Data Analysis. New York: Springer (2009).

28. Hunter LW, Jayachandran M, Miller VM. Sex Differences in the Expression of Cell Adhesion Molecules on Microvesicles Derived From Cultured Human Brain Microvascular Endothelial Cells Treated With Inflammatory and Thrombotic Stimuli. Biol Sex Differ (2019) 10:26. doi: 10.1186/s13293-0190241-y

29. Hiramatsu R, Fukuhara S, Mitsuda S, Yokomichi T, Kataoka T. Betulinic Acid and Oleanolic Acid, Natural Pentacyclic Triterpenoids, Interfere With NLinked Glycan Modifications to Intercellular Adhesion Molecule-1, But Not Its Intracellular Transport to the Cell Surface. Eur J Pharmacol (2015) 767:126-34. doi: 10.1016/j.ejphar.2015.10.017

30. Harada M, Morimoto K, Kondo T, Hiramatsu R, Okina Y, Muko R, et al. Quinacrine Inhibits Icam-1 Transcription by Blocking Dna Binding of the NF- $\mathrm{kb}$ Subunit p65 and Sensitizes Human Lung Adenocarcinoma A549 Cells to TNF- $\alpha$ and the Fas Ligand. Int J Mol Sci (2017) 18:2603. doi: 10.3390/ ijms18122603

31. Giard DJ, Aaronson SA, Todaro GJ, Arnstein P, Kersey JH, Dosik H, et al. In Vitro Cultivation of Human Tumors: Establishment of Cell Lines Derived From a Series of Solid Tumors. J Natl Cancer Inst (1973) 51:1417-23. doi: $10.1093 /$ jnci/51.5.1417 
32. Quail DF, Joyce JA. Microenvironmental Regulation of Tumor Progression and Metastasis. Nat Med (2013) 19:1423-37. doi: 10.1038/nm.3394

33. Kim J, Bae JS. Tumor-Associated Macrophages and Neutrophils in Tumor Microenvironment. Mediators Inflamm (2016) 2016:6058147. doi: 10.1155/ 2016/6058147

34. Komohara Y, Fujiwara Y, Ohnishi K, Takeya M. Tumor-Associated Macrophages: Potential Therapeutic Targets for Anti-Cancer Therapy. Adv Drug Delivery Rev (2016) 99:180-5. doi: 10.1016/j.addr.2015.11.009

35. Chen Y, Wen H, Zhou C, Su Q, Lin Y, Xie Y, et al. TNF-Alpha Derived From M2 Tumor-Associated Macrophages Promotes Epithelial-Mesenchymal Transition and Cancer Stemness Through the Wnt/beta-catenin Pathway in SMMC-7721 Hepatocellular Carcinoma Cells. Exp Cell Res (2019) 378:41-50. doi: 10.1016/j.yexcr.2019.03.005

36. Gimbrone MAJr., Nagel T, Topper JN. Biomechanical Activation: An Emerging Paradigm in Endothelial Adhesion Biology. J Clin Invest (1997) 99:1809-13. doi: 10.1172/JCI119346

37. Pober JS, Sessa WC. Evolving Functions of Endothelial Cells in Inflammation. Nat Rev Immunol (2007) 7:803-15. doi: 10.1038/nri2171

38. Wu F, Liu L, Zhou H. Endothelial Cell Activation in Central Nervous System Inflammation. J Leukoc Biol (2017) 101:1119-32. doi: 10.1189/jlb.3RU0816-352RR

39. Liao JK. Linking Endothelial Dysfunction With Endothelial Cell Activation. J Clin Invest (2013) 123:540-1. doi: 10.1172/JCI66843

40. De Caterina R, Libby P, Peng HB, Thannickal VJ, Rajavashisth TB, Gimbrone MAJr., et al. Nitric Oxide Decreases Cytokine-Induced Endothelial Activation. Nitric Oxide Selectively Reduces Endothelial Expression of Adhesion Molecules and Proinflammatory Cytokines. J Clin Invest (1995) 96:60-8. doi: 10.1172/JCI118074

41. Reina M, Espel E. Role of LFA-1 and ICAM-1 in Cancer. Cancers (Basel) (2017) 9:153. doi: 10.20944/preprints201709.0146.v2

42. Zimmerman AW, Joosten B, Torensma R, Parnes JR, Van Leeuwen FN, Figdor CG. Long-Term Engagement of CD6 and ALCAM is Essential for Tcell Proliferation Induced by Dendritic Cells. Blood (2006) 107:3212-20. doi: 10.1182/blood-2005-09-3881

43. Dhawan A, Friedrichs J, Bonin MV, Bejestani EP, Werner C, Wobus M, et al. Breast Cancer Cells Compete With Hematopoietic Stem and Progenitor Cells for
Intercellular Adhesion Molecule 1-Mediated Binding to the Bone Marrow Microenvironment. Carcinogenesis (2016) 37:759-67. doi: 10.1093/carcin/bgw057 44. Talmadge JE, Fidler IJ. AACR Centennial Series: The Biology of Cancer Metastasis: Historical Perspective. Cancer Res (2010) 70:5649-69. doi: 10.1158/0008-5472.CAN-10-1040

45. Wilhelm I, Molnar J, Fazakas C, Hasko J, Krizbai IA. Role of the Blood-Brain Barrier in the Formation of Brain Metastases. Int J Mol Sci (2013) 14:1383411. doi: 10.3390/ijms14011383

46. Brabletz S, Schmalhofer O, Brabletz T. Gastrointestinal Stem Cells in Development and Cancer. J Pathol (2009) 217:307-17. doi: 10.1002/path.2475

47. Bos PD, Zhang XH, Nadal C, Shu W, Gomis RR, Nguyen DX, et al. Genes That Mediate Breast Cancer Metastasis to the Brain. Nature (2009) 459:10059. doi: 10.1038/nature08021

48. Yao H, Price TT, Cantelli G, Ngo B, Warner MJ, Olivere L, et al. Leukaemia Hijacks a Neural Mechanism to Invade the Central Nervous System. Nature (2018) 560:55-60. doi: 10.1038/s41586-018-0342-5

49. Mcsherry EA, Mcgee SF, Jirstrom K, Doyle EM, Brennan DJ, Landberg G, et al. JAM-a Expression Positively Correlates With Poor Prognosis in Breast Cancer Patients. Int J Cancer (2009) 125:1343-51. doi: 10.1002/ijc.24498

50. Naik MU, Naik TU, Suckow AT, Duncan MK, Naik UP. Attenuation of Junctional Adhesion Molecule-A Is a Contributing Factor for Breast Cancer Cell Invasion. Cancer Res (2008) 68:2194-203. doi: 10.1158/0008-5472.CAN07-3057

Conflict of Interest: The authors declare that the research was conducted in the absence of any commercial or financial relationships that could be construed as a potential conflict of interest.

Copyright (C) 2021 Wang, Dong, Higazy, Jin, Zou, Chen, Inayat, Hu and Cui. This is an open-access article distributed under the terms of the Creative Commons Attribution License (CC BY). The use, distribution or reproduction in other forums is permitted, provided the original author(s) and the copyright owner(s) are credited and that the original publication in this journal is cited, in accordance with accepted academic practice. No use, distribution or reproduction is permitted which does not comply with these terms. 